\title{
Chromatin reorganization on a 'mega' scale
}

Fusion oncogenes of NUT (also known as NUTM1) with several different genes, including bromodomaincontaining protein 3 (BRD3), BRD4 and NSD3, cause the aggressive squamous cell cancer NUT midline carcinoma (NMC). These gene fusions result in the tethering of the NUT protein, which contains a putative transcriptional activation domain, to acetylated chromatin via either direct or indirect association with a bromodomain and extra terminal (BET) protein (either BRD3 or BRD4). Through analysing the genomic loci with which the most common NUT fusion protein, BRD4-NUT, associates, Alekseyenko, Walsh et al. have discovered that the NUT fusions localize to very large hyperacetylated domains within chromatin, which they term 'megadomains', leading to aberrant transcriptional programmes that promote tumorigenesis.

BRD4-NUT has been previously shown to localize in 80-100 discrete nuclear foci within NMC cells. The authors confirmed this and showed that overexpression of BRD4-NUT in non-NMC 293T cells is sufficient to form these foci. Chromatin immunoprecipitation followed by sequencing (ChIP-seq) showed that BRD4-NUT localized in very large megadomains, which differ from recently described 'super-enhancers' in that megadomains are an order of magnitude larger $(100 \mathrm{~kb}-2 \mathrm{Mb})$ and have a more continuously enriched profile of histone $\mathrm{H} 3$ lysine 27 acetylation (H3K27ac) rather than clusters of $\mathrm{H} 3 \mathrm{~K} 27 \mathrm{ac}$ peaks. These megadomains were associated with increased levels of gene and non-coding RNA (ncRNA) transcription.
Localization of BRD4-NUT to $\mathrm{H} 3 \mathrm{~K} 27 \mathrm{ac}$-enriched megadomains was also observed in NMC cells that endogenously expressed the fusion (TC-797 cells), and transcription from these domains decreased when cells were treated with the BET inhibitor JQ1. Although there was little overlap in the genomic regions bound in TC-797 cells and 293T cells overexpressing BRD4-NUT, there was overlap among different BRD4-NUT ${ }^{+} \mathrm{NMC}$ cell lines. ChIP-seq time-course analyses in 293T cells overexpressing inducible BRD4-NUT indicated that there is a gradual spread of BRD4-NUT and H3K27ac from narrow 'seed' regions on existing enhancers (interestingly, super-enhancers are not over-represented in the seed regions) into megadomains, the boundaries of which are marked by topologically associating domains (TADs). Together, these data suggest that the lineage of the cell in which BRD4-NUT is expressed determines the location of megadomains, thereby influencing gene transcription.

The authors reasoned that megadomains shared among different NMC cells might contain crucial oncogenic regions. They identified a group of 33 genes that were expressed in 3 of 4 NMC cell lines and whose expression was knocked down by JQ1 treatment. These included several long ncRNAs (lncRNAs), TP63 and the Mediator subunit MED24. Knockdown of the lncRNA PVT1 (which increases MYC levels) or of MED24 in TC-797 cells induced differentiation, and knockdown of TP63 reduced TC-797 cell viability. Therefore, it seems that the widespread epigenetic changes induced by NUT fusions can lead to the activation of multiple genes that can in turn block differentiation or maintain growth of NMC. This might account for the short evolution time and aggressiveness of NMC.

Sarah Seton-Rogers

ORIGINAL RESEARCH PAPER Alekseyenko, A. A., Walsh, E. M. et al. The oncogenic BRD4-NUT chromatin regulator drives aberrant transcription within large topological domains. Genes Dev. 29, 1507-1523 (2015)

FURTHER READING French, C. NUT midline carcinoma. Nat. Rev. Cancer 14, 149-150 (2014)

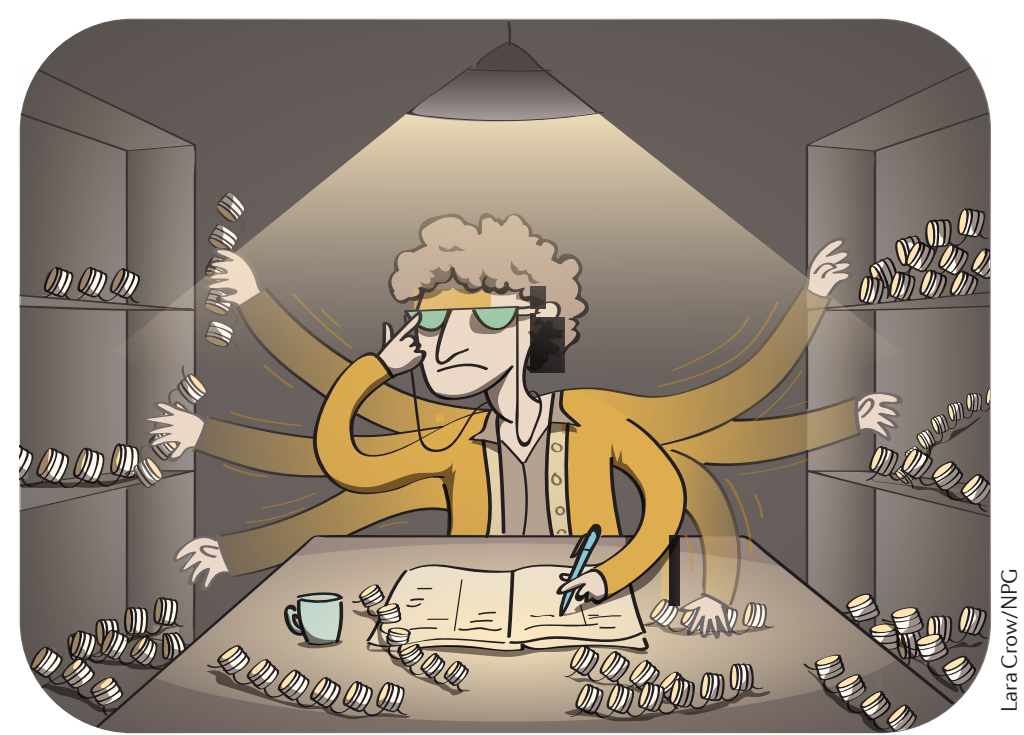

